ARTICLE

\title{
Emotion-based brain mechanisms and predictors for SSRI and CBT treatment of anxiety and depression: a randomized trial
}

Stephanie M. Gorka ${ }^{1}$, Christina B. Young ${ }^{2}$, Heide Klumpp ${ }^{1,3}$, Amy E. Kennedy ${ }^{1}$, Jennifer Francis ${ }^{1}$, Olusola Ajilore $\mathbb{D}^{1}$, Scott A. Langenecker ${ }^{1,3}$, Stewart A. Shankman (iD ${ }^{1,3}$, Michelle G. Craske ${ }^{4}$, Murray B. Stein ${ }^{5}{ }^{5}$ and K. Luan Phan ${ }^{1,3,6}$

Mechanisms and predictors for the successful treatment of anxiety and depression have been elusive, limiting the effectiveness of existing treatments and curtailing the development of new interventions. In this study, we evaluated the utility of three widely used neural probes of emotion (experience, regulation, and perception) in their ability to predict symptom improvement and correlate with symptom change following two first-line treatments-selective serotonin reuptake inhibitors (SSRIs) and cognitive-behavioral therapy (CBT). Fifty-five treatment-seeking adults with anxiety and/or depression were randomized to 12 weeks of SSRI or CBT treatment (ClinicalTrials.gov identifier: NCT01903447). Functional magnetic resonance imaging (fMRI) was used to examine frontolimbic brain function during emotion experience, regulation, and perception, as probed by the Emotion Regulation Task (ERT; emotion experience and regulation) and emotional face assessment task (EFAT; emotion perception). Brain function was then related to anxiety and depression symptom change. Results showed that both SSRI and CBT treatments similarly attenuated insula and amygdala activity during emotion perception, and greater treatment-related decrease in insula and amygdala activity was correlated with greater reduction in anxiety symptoms. Both treatments also reduced amygdala activity during emotion experience but brain change did not correlate with symptom change. Lastly, greater pre-treatment insula and amygdala activity during emotion perception predicted greater anxiety and depression symptom improvement. Thus, limbic activity during emotion perception is reduced by both SSRI and CBT treatments, and predicts anxiety and depression symptom improvement. Critically, neural reactivity during emotion perception may be a non-treatment-specific mechanism for symptom improvement.

Neuropsychopharmacology (2019) 44:1639-1648; https://doi.org/10.1038/s41386-019-0407-7

\section{INTRODUCTION}

Internalizing psychopathologies involving anxiety and depression are highly prevalent and carry substantial burden [1]. Two goldstandard internalizing disorder treatments-selective serotonin reuptake inhibitor (SSRI) pharmacotherapy and cognitive behavioral therapy (CBT) - are equally effective in reducing anxiety and depression symptoms [2,3]. However, treatment response to either strategy is heterogeneous and a large proportion of patients remain symptomatic after an initial trial $[4,5]$. Inadequate clinical outcomes have been attributed to lack of attention to 'how' and 'for whom' an intervention works. There are also few validated, biological predictors to guide the patient to the treatment with the highest likelihood of success for that individual. The identification of internalizing psychopathology treatment predictors, and a better understanding of treatment mechanisms, has the potential to drastically improve patient care by advancing precision medicine and treatment development based on brain-based mechanisms.

Proposed putative targets of internalizing psychopathology treatments are the behavioral-brain mechanisms underlying emotion processing, including sustained experience of negative affective states (i.e., emotion experience), identification of socioemotional information (i.e., emotion perception), and volitional emotion regulation capabilities (i.e., emotion regulation).

Importantly, these emotion processes, as probed by specific fMRI tasks, are believed to reflect the primary dysfunctions shared across internalizing psychopathologies (e.g., aberrant biases towards negative stimuli, impaired regulation of negative affect) [6]. Nearly three decades of functional neuroimaging research have demonstrated that these three aspects of emotion processing are instantiated by a frontolimbic neural circuit comprised of a discrete set of regions including the amygdala, anterior insula (aINS), and subdivisions of the prefrontal cortex (PFC), such as the dorsomedial, dorsolateral, ventromedial, and ventrolateral PFC (dmPFC, dIPFC, vmPFC, vIPFC) [7, 8]. In comparison to healthy individuals, those with anxiety and depression exhibit increased amygdala and aINS reactivity to negatively valenced stimuli, as well as decreased dIPFC and dmPFC activity during the employment of effortful emotion regulation strategies such as cognitive reappraisal $[8,9]$. A few studies have also shown that anxiety and depression symptoms correlate with activity in these areas during emotion perception [10].

\footnotetext{
${ }^{1}$ Department of Psychiatry, University of Illinois at Chicago, Chicago, IL, USA; ${ }^{2}$ Department of Psychiatry and Behavioral Sciences, Stanford University, Stanford, CA, USA; ${ }^{3}$ Department of Psychology, University of Illinois at Chicago, Chicago, IL, USA; ${ }^{4}$ Department of Psychology, University of California-Los Angeles, Los Angeles, CA, USA; ${ }^{5}$ Departments of Psychiatry and Family Medicine and Public Health, University of California-San Diego, La Jolla, CA, USA and ${ }^{6}$ Mental Health Service Line, Jesse Brown Medical Center, Chicago, IL, USA

Correspondence: Stephanie M. Gorka (sgorka2@uic.edu)

These authors contributed equally: Stephanie M. Gorka, Christina B. Young
}

Received: 14 January 2019 Revised: 26 April 2019 Accepted: 29 April 2019

Published online: 6 May 2019 
1640

It stands to reason that effective treatments may modify these same frontolimbic areas, and that neural markers exist to predict treatment response. In accord, several studies examining either SSRI or CBT in specific diagnostic groups have found that successful treatment decreases limbic neural responsivity to aversive stimuli and enhances PFC functioning [11-14]. Previous research also suggests that emotion-based neuroimaging measures may be useful for predicting treatment response. Greater pre-treatment PFC and anterior cingulate cortex (ACC) activity has been shown to predict symptom improvement in CBT or SSRI across several tasks and internalizing psychopathologies $[13,15,16]$. Limbic regions such as the amygdala and aINS have also been identified as potential treatment predictors though findings have been somewhat inconsistent $[17,18]$. It is important to note that SSRI and CBT literatures have produced similar findings within certain emotional processes, but studies have primarily used one probe at a time. Several emotion processes have rarely been studied simultaneously, and it is unclear if and how these treatments differ in their neural mechanisms of action and predictors. Limitations of prior work also include a focus on individual diagnoses (one disorder at a time), and utilization of a single task of emotion function rather than complementary probes of different aspects of emotion processing.

To address these gaps, the current study asked two main questions: (1) are neural measures of emotion processing sensitive to SSRI and/or CBT treatment (i.e., do they change with treatment?); and (2) does pre-treatment neural activity during emotion processing predict SSRI and/or CBT response? Our aim was to define an improvement-related biomarker and/or a treatment-specific biomarker. Treatment-seeking adults with a range of depressive and anxiety disorders were enrolled and randomized to either 12 weeks of SSRI or CBT treatment. Frontolimbic circuit function was assessed using well-validated tasks of emotion experience, regulation, and perception before and after treatment during functional magnetic resonance imaging (fMRI). It is important to highlight that studies of treatment mechanisms and predictors can utilize different designs, including comparisons between active treatments and placebo. The current study opted not to include a placebo given that it would have significantly hindered feasibility by requiring inclusion of both a psychotherapy and medication control arm, and there would have been a reduced number of patients willing to enroll in the study. Nevertheless, the current design is similar to several other published treatment outcome studies (e.g., [19]).

\section{METHODS}

Participants

The current study was designed to be consistent with, and funded by the NIMH Research Domain Criteria (RDoC) Initiative (RFA-MH13-080). Treatment-seeking individuals with a range of internalizing psychopathologies and symptoms were recruited via flyers and advertisements posted in the Chicago community and local psychiatric clinics. Individuals were enrolled if they met fullthreshold or sub-threshold criteria (all but one criterion for a specific disorder) for at least one DSM-5 depressive or anxiety disorder, reported a total score of $\geq 23$ on the Depression, Anxiety, and Stress Scale (DASS-21 [20]), had a Global Assessment of Functioning score of $\leq 60$, and were between the ages of 18 and 65 years. Exclusion criteria are detailed in Supplementary Materials. Procedures were approved by the UIC Institutional Review Board, and informed consent was obtained from all participants. The study was a parallel group randomized control trial with 1:1 allocation ratio and registered in ClinicalTrials.gov (Identifier: NCT01903447). All participants were monetarily compensated for their time.

The CONSORT diagram illustrates the recruitment, intervention allocation, follow-up, and data-analysis flow (Fig. 1), resulting in 55 participants (28 SSRI, 27 CBT) with both pre-treatment and posttreatment $\mathrm{fMRI}$ data included for treatment analyses.

Internalizing psychopathology assessment

The SCID-5 [21] was used to determine current and lifetime Axis I diagnoses. Eligibility and principal diagnosis warranting treatment were determined by a consensus panel consisting of at least three trained clinicians or study staff. At both pre-treatment and posttreatment, the Hamilton Rating Scale for Depression (HAMD) [22] assessed depression symptom severity, and the Hamilton Rating Scale for Anxiety (HAMA) [23] assessed anxiety symptom severity. HAMD and HAMA total scores served as the primary outcome measures. In addition, given recent studies showing that treatments may differentially impact certain types of anxiety symptoms [24], we calculated the HAMA psychic anxiety (items $1-6,14)$ and somatic anxiety (items 7-13) subscales. Depression and anxiety treatment response were defined as $\geq 50 \%$ symptom improvement on the HAMD and HAMA total, respectively.

Treatment procedures

Participants were randomized to either 12 weeks of SSRI or CBT treatment, as previously detailed $[25,26]$. SSRI and CBT treatment details are described in Supplementary Materials.

Brain and behavioral tasks

Emotional regulation task (ERT). The ERT is a well-validated task to probe frontolimbic responses during sustained negative emotional experience and its volitional regulation using cognitive reappraisal [8]. In a block-design, participants viewed negative and neutral images from International Affective Picture System (IAPS) under three conditions: (1) "Look Neutral" by naturally viewing neutral images without attempting to change one's emotional response; (2) "Look Negative" by naturally viewing negative images without attempting to change one's emotional response; and (3) "Reappraise Negative" (negative images) by interpreting the depicted scenario in a less negative way to reduce negative affect evoked by the aversive images. At the end of each of the four task blocks, participants rated how negative they felt on a 5point scale ( $1=$ not at all, $5=$ extremely) using a button response.

Emotional face assessment task (EFAT). The EFAT is a wellvalidated task to probe limbic responses during the identification of socio-emotional information (i.e., emotion type based on facial expression), labeled emotion perception [27]. In a block-design, participants viewed and matched Emotional Faces or geometric Shapes. In this task, trios of faces were presented, and participants were instructed to select one of the two bottom faces that expressed the same emotion (angry, fearful, happy, sad) as the target face on top; or to select one of the two bottom shapes that matched the same shape (circles, rectangles, triangles). Accuracy and reaction times (RT) to button press for each trial were also recorded.

fMRI data acquisition, preprocessing, and analyses MR scan parameters, preprocessing steps, and analyses are described in Supplementary Materials. For ERT, we created two contrasts: Look Negative vs. Look Neutral to capture negative emotional experience and Reappraise Negative vs. Look Negative to capture emotion regulation. For EFAT, we created one contrast, Emotional Faces vs. Shapes, to assess emotion perception.

Data analysis plan

Treatment effects on brain function. The main effect of Time, and the Arm (SSRI, CBT) $\times$ Time interaction were examined using mixed ANOVAs with Arm as the between-subject factor, and Time and Condition (i.e., Look Neutral vs. Look Negative [Experience], Reappraise Negative vs. Look Negative [Regulation], or Emotional Faces vs. Shapes [Perception]) as within-subject factors. To control 


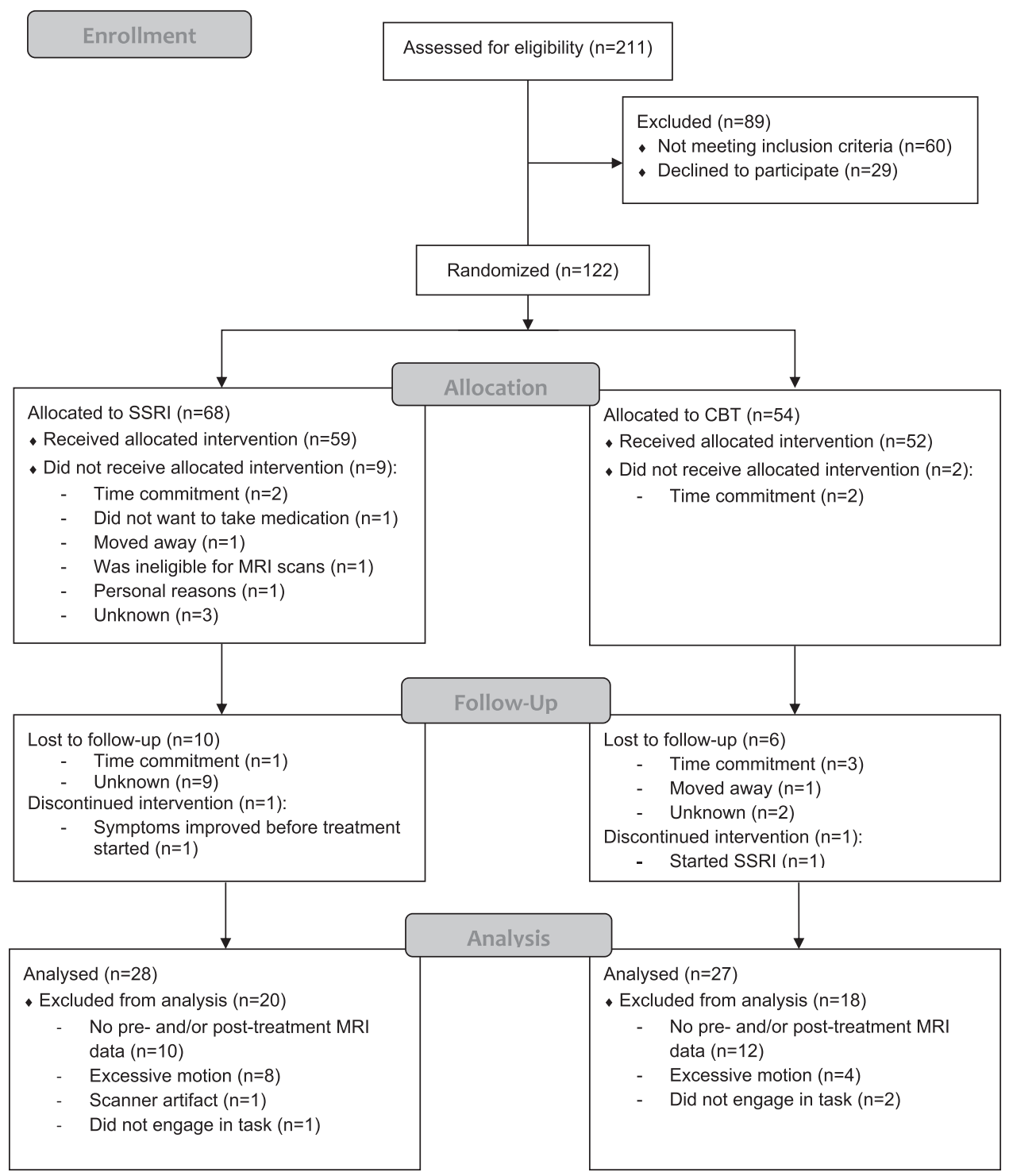

Fig. 1 CONSORT flow diagram. Flow diagram showing the progress of participants throughout the trial. SSRIselective serotonin reuptake inhibitors, CBTcognitive behavioral therapy

for Type I error inflation, omnibus $F$ statistics for the main effect of Time and the Arm $\times$ Time interaction effect were subjected to whole-brain voxel-wise false discovery rate (FDR) correction $(p<$ 0.05 ) with an extent threshold of 50 voxels.

A complementary region-of-interest (ROI) approach was used to examine the effect of treatment in selective regions specified a priori (amygdala, insula, dmPFC, dIPFC, vIPFC), which were significantly activated by our two tasks. Activation of these areas was confirmed from ERT and EFAT contrasts (Look Negative vs. Look Neutral: amygdala; Reappraise Negative vs. Look Negative: dmPFC, dIPFC, vIPFC; Emotional Faces vs. Shapes: amygdala, insula) using whole-brain voxel-wise FDR correction $(p<0.05)$ with an extent threshold of 50 voxels amongst all participants with pretreatment ERT and EFAT data $(n=93)$. Then, for participants who completed treatment, parameter estimates were extracted at both time points using anatomical ROls. We chose to use anatomical ROIs (rather than task-based spheres surrounding peak foci) in order to standardize our approach in such a way that other research and clinical groups could easily use the same procedures. The amygdala and insula masks were defined via the AAL atlas and created using MARINA (http://www.bion.de/Marina.html). Bilateral amygdala ROls were extracted from ERT Look Neutral vs. Look Negative and EFAT Emotional Faces vs. Shapes. A bilateral insula ROI was extracted from EFAT Emotional Faces vs. Shapes. For the PFC regions, there are no widely accepted dmPFC, dIPFC, or vIPFC anatomical masks, to our knowledge. Therefore, we used Neurosynth, a platform for term-based meta-analysis of fMRI data [28], using the search terms "dorsomedial prefrontal," "dorsolateral prefrontal," and "ventrolateral prefrontal," respectively. ROls were created from thresholded association test maps of each search term. The dmPFC and dIPFC association test maps were thresholded using a height threshold of $z>5$ and an extent threshold of 150 voxels; the vIPFC association test map used the same extent threshold and a height threshold of $z>4$.

Treatment-related brain-clinical relationships. To test for a relationship between change in brain function and change in clinical symptoms, we calculated pre-treatment to posttreatment change scores for our brain and symptom measures (Time 1 [T1] vs. Time 2 [T2]). For brain measures we calculated change in extracted parameter estimates of activation. For symptom measures we calculated change in HAMD and HAMA total scores and both HAMA subscales. The percent change scores for brain and depression and anxiety symptoms were 
1642

then entered into a non-parametric correlation analysis using Spearman's Rho.

Treatment prediction. The association between pre-treatment brain activity and treatment outcomes was examined using T1 extracted parameter estimates from the a priori anatomical ROls described above. We specifically conducted a series of hierarchical linear regression analyses with Arm and T1 ROI activation entered in Step 1 and the interaction of Arm and T1 ROI activation entered in Step 2. Percent change in HAMD and HAMA total scores, and both HAMA subscales, were included as dependent variables.

\section{RESULTS}

Demographic and clinical characteristics

There were no significant differences in demographic and clinical characteristics between participants randomized to SSRI or CBT treatment (Table 1). HAMD and HAMA total scores and HAMA subscales significantly decreased pre-treatment to post-treatment for both arms, all $p s<0.001$, and the extent of symptom reduction did not significantly differ based on treatment modality, all $p s>$ 0.56 . Treatment response for depression $(\mathrm{SSRI}=67.86 \%$; $\mathrm{CBT}=$
$74.07 \%)$ and anxiety (SSRI $=71.43 \%$; $C B T=81.48 \%$ ) did not differ significantly between arms, all $p s>0.57$.

Of the individuals randomized to treatment, there were no differences in sex, race, or baseline HAMD or HAMA symptoms between individuals who did and did not complete all 12 weeks of treatment, $p s>0.61$. However, individuals who completed treatment were significantly older than individuals who dropped out of treatment, $F(1,121)=6.07, p<0.05$. There were no differences between those who completed versus dropped out of treatment in baseline neural activation, based on the extracted ROI parameter estimates from the EFAT and ERT task effects, $p s>0.54$.

Treatment effects on brain function

Whole-brain search. In a whole-brain voxel-wise search, there were no significant main effects of Arm or Time, and no significant Arm $\times$ Time interaction.

ROI-specific search. Details regarding regions that were activated by the tasks at the whole-brain level are presented in Supplementary Materials. Examination of changes in EFAT activation as a function of treatment revealed a significant main effect of Time in insula and amygdala such that activation was

Table 1. Demographic and clinical characteristics of participants randomized to selective serotonin reuptake inhibitors (SSRI) and cognitive behavioral therapy (CBT) treatments

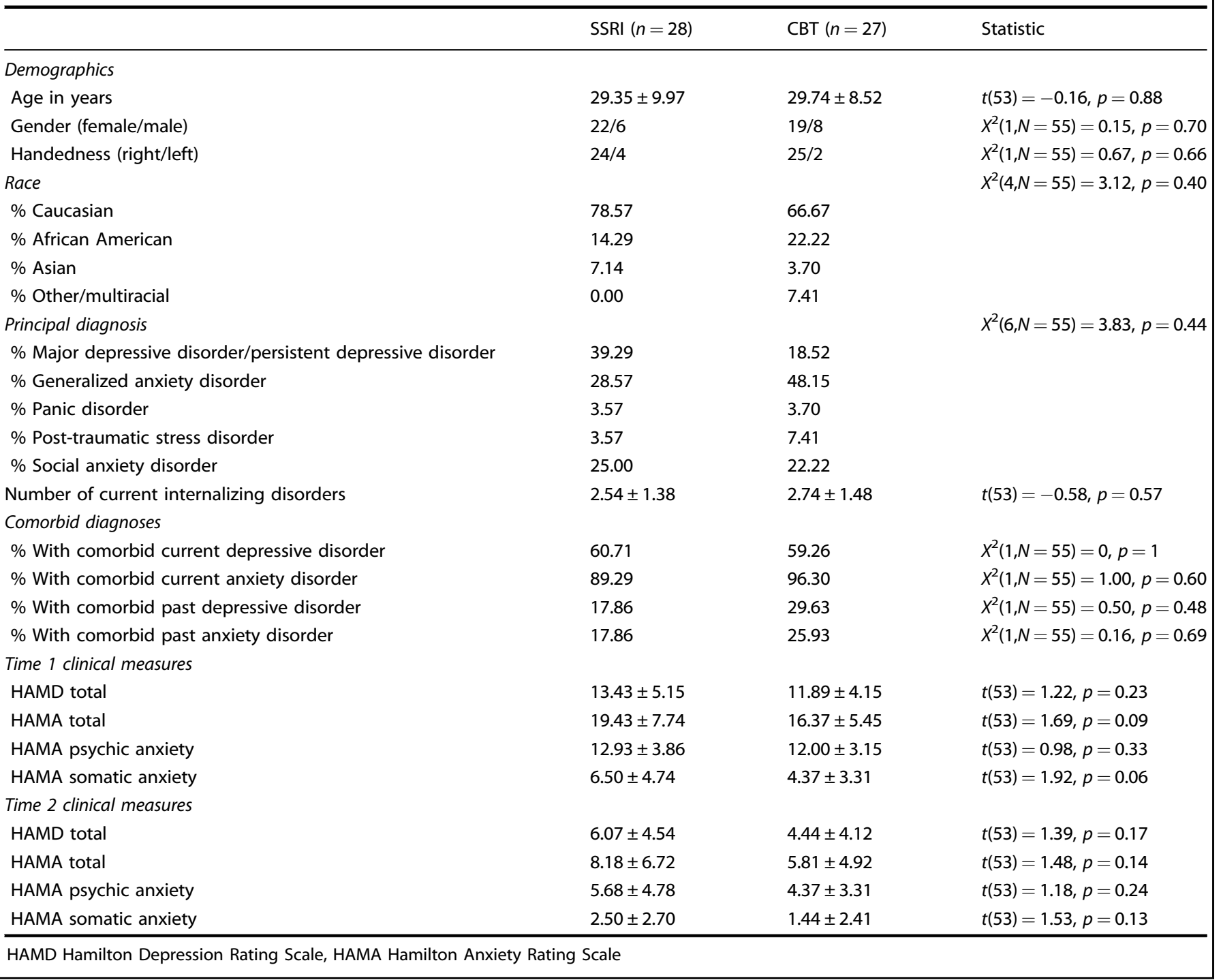


reduced during emotion perception pre-to-post treatment (Table 2, Fig. 2). For the ERT task, results indicated that there was also a main effect of Time in the amygdala during emotion experience (reduced activation; Table 2, Fig. 2). There were no other significant main effects of Time or Arm, and no significant Arm $\times$ Time interactions in any other ROI.

\section{Treatment-related brain-clinical relationships}

Greater symptom improvement in HAMA total scores were related to greater decreases in insula activation, $r_{\mathrm{s}}=0.28, p=0.04$, and amygdala activation, $r_{\mathrm{s}}=0.37, p=0.01$, during EFAT emotion perception (Fig. 3a). With regard to HAMA subscales, greater symptom improvement in HAMA psychic symptoms was associated with greater decreases in insula activation, $r=0.28, p=$ 0.04, and amygdala activation, $r=0.34, p=0.01$, during EFAT emotion perception. There were no associations with change in HAMA somatic symptoms and change in brain activation, $p s>$ 0.15 . In addition, no other brain regions showed a significant relation with symptom improvement, all $p s>0.14$.

\section{Treatment prediction}

The results of the treatment prediction models for HAMD and HAMA total scores are presented in Table 2. The treatment prediction models for the HAMA subscales are presented in Supplementary Materials (Table S1). Greater pre-treatment insula and amygdala activation during emotion perception was associated with greater HAMA and HAMD total symptom improvement for both SSRI and CBT treatment arms (Fig. 3b). Greater pretreatment insula and amygdala activation during emotion perception was associated with greater HAMA psychic anxiety symptom improvement across arms but not HAMA somatic anxiety symptom improvement. There were no other significant main effects or $\mathrm{ROI} \times$ Arm interactions.

\section{DISCUSSION}

The current study made several broad observations. First, the effect of treatment on emotion brain function was relatively focal, and had the biggest impact on limbic reactivity during emotional stimuli. Second, change in emotion-based markers of brain function did not differentiate the effect of SSRI from CBT. Third, baseline/pre-treatment brain function explained some of the variance in treatment success but did not differentiate treatment responses. Fourth, the observable significance of a treatment effect on brain change and brain prediction was specific to certain emotional processes (i.e., emotion perception). More specifically, we found across treatment arms, insula and amygdala reactivity during emotion perception, and amygdala reactivity during emotion experience, decreased pre-treatment to post-treatment. Greater decreases in insula and amygdala reactivity during emotion perception (only) were related to greater anxiety symptom improvement. We also found that greater pretreatment insula and amygdala activation during emotion perception was associated with greater anxiety and depression symptom improvement across treatment arms.

Our results suggest that both treatments were associated with decreases in limbic activation in response to emotional stimuli. Reductions in insula and amygdala reactivity during emotion perception, in particular, are potential mechanisms of anxiety symptom improvement since activation in both regions decreased pre-to-post treatment, and changes in brain activity during emotion perception correlated with the extent of symptom change. Notably, examination of HAMA subscales revealed that neural activation patterns were specifically associated with change in HAMA psychic symptoms, but not HAMA somatic symptoms. Although amygdala reactivity during emotion experience also decreased pre-to-post treatment, change in brain activity during this condition did not relate to symptom change. This set of findings is important, and consistent with several theories and studies suggesting that effective internalizing psychopathology treatments dampen exaggerated responding to emotion cues [29-31]. Our results therefore converge with existing literature while demonstrating for the first time that limbic function during emotion perception may be a broad, non-specific neural mechanism of effective psychic anxiety-based treatment approaches. The findings also demonstrate that treatment effects were especially robust in the insula during emotion perception (large effect size; $\eta_{\mathrm{G}}{ }^{2}=0.45$ ), which is important considering the insula mediates interactions between large-scale brain networks in order to generate internal bodily states and guide behavior [3234]. The insula is therefore a viable internalizing disorder treatment mechanism.

In addition to treatment change, our results indicate that insula and amygdala activation during emotion perception predicts treatment response as greater pre-treatment reactivity in these two regions was associated with greater symptom improvement following SSRI and CBT. The insula and amygdala are key nodes of the frontolimbic circuit and are strongly implicated in the pathophysiology of internalizing psychopathology [35]. They have also been shown to predict internalizing psychopathology treatment response in numerous studies involving either CBT or SSRI; however, the direction of pre-treatment activation has been somewhat inconsistent with studies reporting both hyperactivity and hypoactivity as a positive prognostic indicator [36, 37]. These discrepancies are likely related to differences in $\mathrm{fMRI}$ paradigms and internalizing psychopathology patient characteristics (e.g., primary MDD versus anxiety disorder, extent of comorbid psychopathology). Our findings importantly add to this literature and suggest that in a treatment-seeking, highly comorbid patient population, internalizing psychopathology treatments may be most useful for those with increased baseline limbic reactivity to socio-emotional faces. As others have speculated, individuals with enhanced baseline emotion processing may have the most to gain from treatment, and therefore experience the most symptom improvement [17]. Along these lines, through pharmacological (SSRIs) and learning (CBT) processes, effective treatments may directly engage and 'normalize' amygdala and insula hyperactivity resulting in the greatest benefit for individuals with this particular profile. It is also possible that individuals with greater insula and amygdala reactivity at baseline have a more intact mechanism for discriminating between negative and neutral stimuli and this capability "sets the stage" for greater symptom relief and improved treatment outcomes $[36,38]$.

Contrary to hypotheses, our neural measures of emotion regulation did not change with treatment nor predict symptom improvement. This is in contrast to a few other studies that have found that internalizing psychopathology treatments impact emotion regulation probes, including a prior study by our group demonstrating in a sample of veterans with PTSD that PFC activation during emotion regulation increases with SSRI treatment and predicts PTSD symptom improvement [39]. The discrepancy between findings may be related to internalizing psychopathology patient characteristics (e.g., veterans with PTSD versus treatment-seeking community population). It is also important to highlight that there was substantial variability in change in PFC activation pre-to-post treatment in our sample and in larger samples, it may be possible to identify certain subgroups of individuals where PFC activation during emotion regulation changes with treatment and/or predicts symptom improvement. The current results nevertheless highlight that across all subjects, in a typical IP clinic setting, tasks that probe neural markers of emotion regulation may be less useful in identifying mechanisms or predictors of response. Emotion perception, particularly neural reactivity to socioemotional faces captured via the EFAT task, appears to be a more widely applicable, clinically useful tool in this regard. 


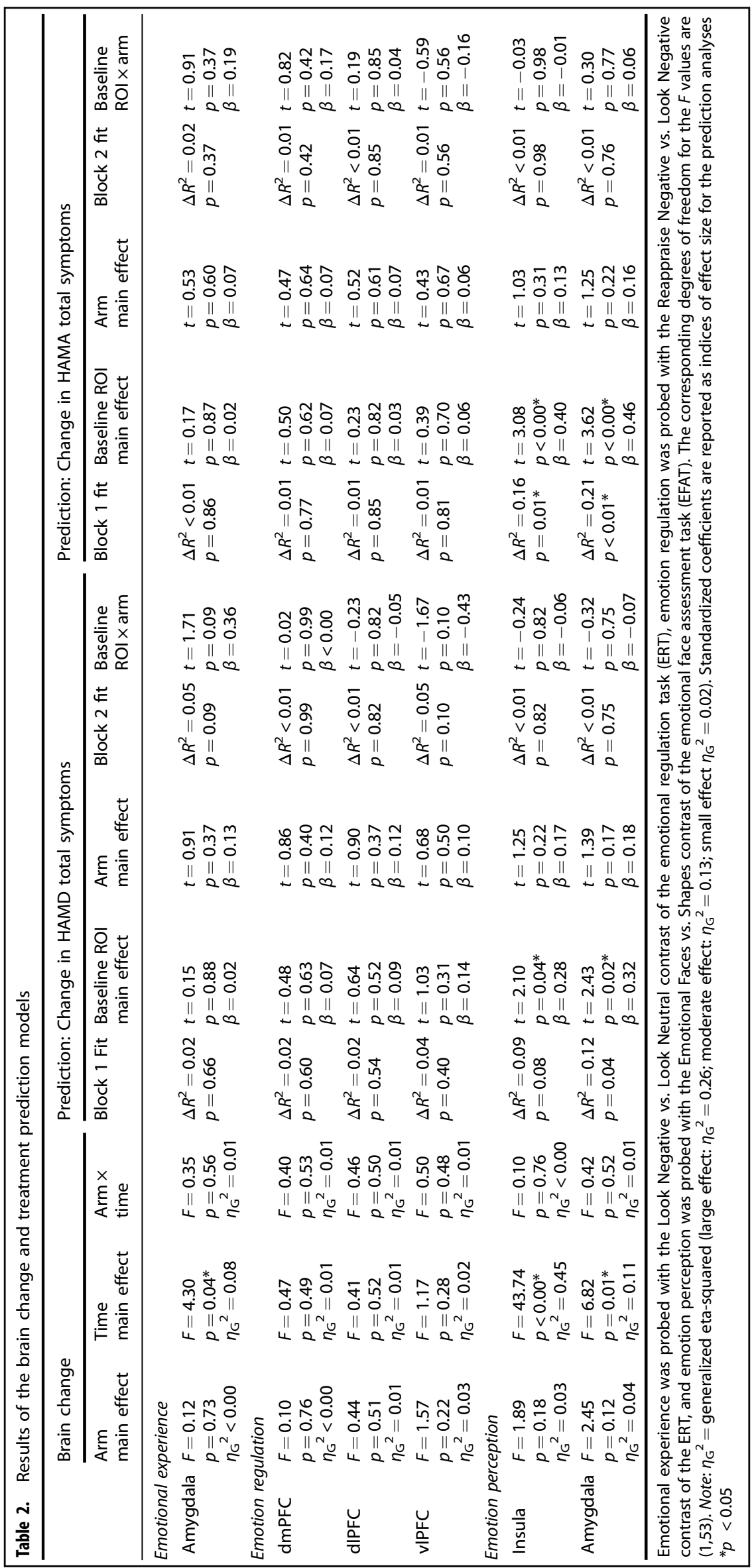


Emotional Experience: Amygdala *
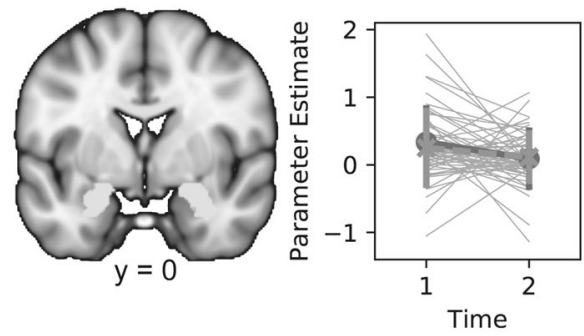

Emotional Regulation: dIPFC
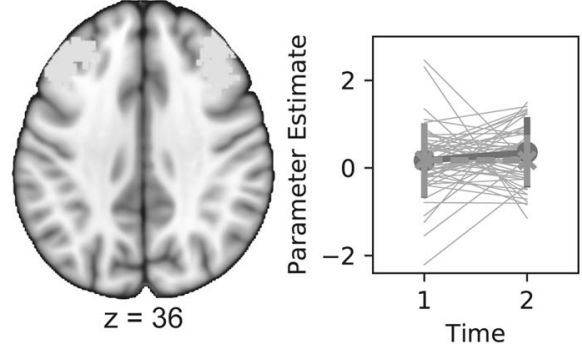

Emotion Perception: Insula *
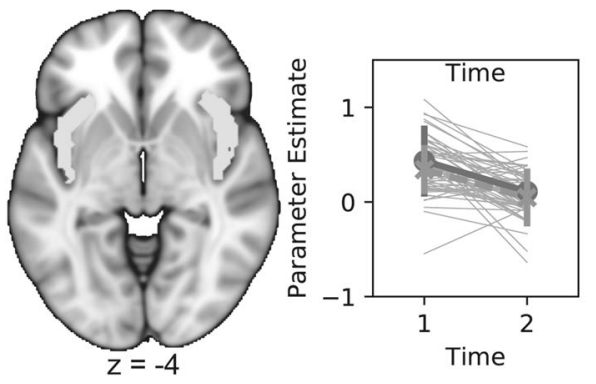

Treatment Arm

\section{Emotional Regulation: dmPFC}
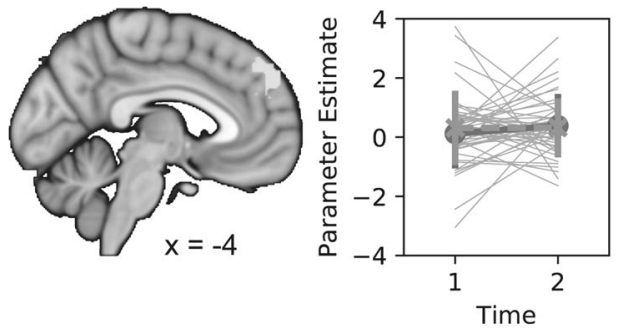

Emotional Regulation: vIPFC

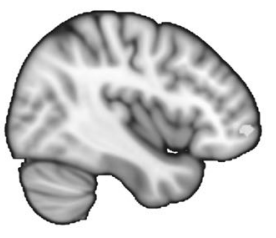

$x=42$

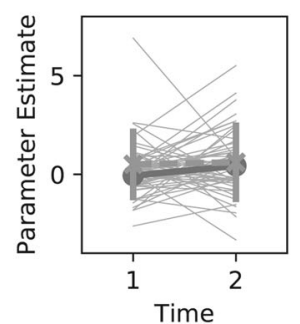

Emotion Perception: Amygdala *
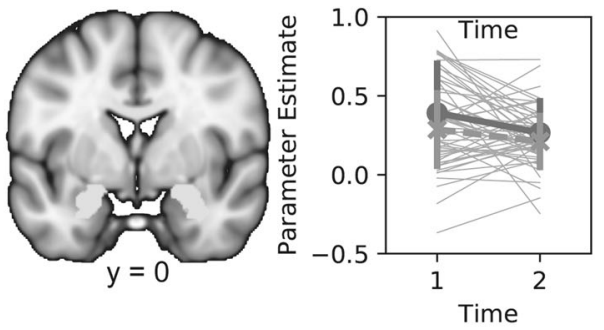

SSRI $(n=28)$

$=*-\operatorname{CBT}(n=27)$

Fig. 2 Change in brain activation during emotion perception, experience, and regulation pre-to-post treatment. Brain images depict anatomical amygdala, insula, and prefrontal cortex masks used for region-of-interest (ROI) analyses. Line graphs illustrate participant-level changes in activation (gray), as well as mean activation ( \pm standard deviation) pre-treatment and post-treatment for selective serotonin reuptake inhibitors (SSRI; blue) and cognitive behavioral therapy (CBT; red) treatment arms. Notes: dmPFC dorsomedial prefrontal cortex, dIPFC dorsolateral prefrontal cortex, vIPFC ventrolateral prefrontal cortex. Emotion experience and emotion regulation were evoked by the emotional regulation task (ERT). Emotion perception was evoked by the emotional face assessment task (EFAT). *Significant change over time, $p<0.05$

We also initially hypothesized that CBT and SSRIs would have similar and distinct treatment mechanisms and predictors. Our results indicated that brain effects and predictors are shared, and not specific to one treatment. Though contrary to our hypotheses, other studies have also failed to identify treatment-specific effects, challenging the model that psychotherapy targets prefrontal 'top down' functions, whereas SSRIs target 'bottom-up' limbic areas [40]. Our findings are more consistent with the broad notion that these two (and perhaps other) internalizing disorder treatments have more in common than not, and therefore share mechanisms and predictors [2]. This conclusion does not preclude the fact that individual differences are still likely important, and that there may be specific 'biotypes' that can help dictate internalizing psychopathology treatment assignment. A point of emphasis moving forward should therefore be person-specific treatment prediction and change. With regard to the current findings, if validated, a subgroup exhibiting high limbic reactivity to socio-emotional stimuli could be assigned to SSRI or CBT, depending entirely on patient preference and/or treatment access.
It is important to highlight that although the current study was transdiagnostic and symptoms were measured continuously, the HAMD and HAMA scales capture relatively narrow dimensions of depression and anxiety symptoms. Numerous large-scale, factoranalytic studies have shown that depression and anxiety exist within a broader hierarchical structure of psychopathology and that there are several higher-order factors (such as fear and distress, and the broader general factor of psychopathology) that may be more closely linked to the etiology of mental illness and perhaps, treatment response (see ref. [41] for a discussion). The current study did not include higher-order measures of internalizing psychopathology such as negative emotionality, fear, and/or distress. Thus, in-order to complement the findings from the current study and advance the overall mission of $\mathrm{RDoC}$, it will be important for future research to include a unifying internalizing disorder measure to continue to elucidate brain mechanisms and predictors of treatment response.

The study produced encouraging results but has several limitations to consider. First, there was no placebo or control 


\section{A. Brain-Clinical Relationships}

\section{Emotion Perception: Insula}

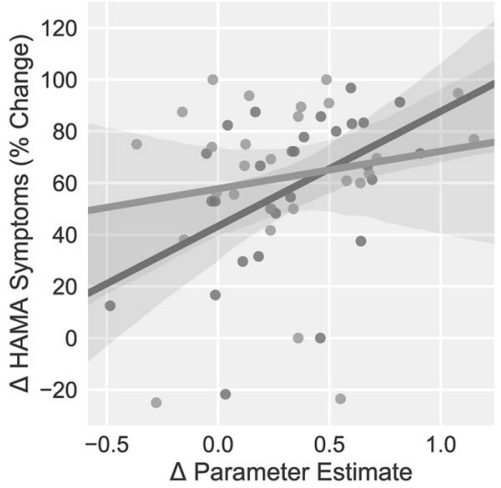

Emotion Perception: Amygdala

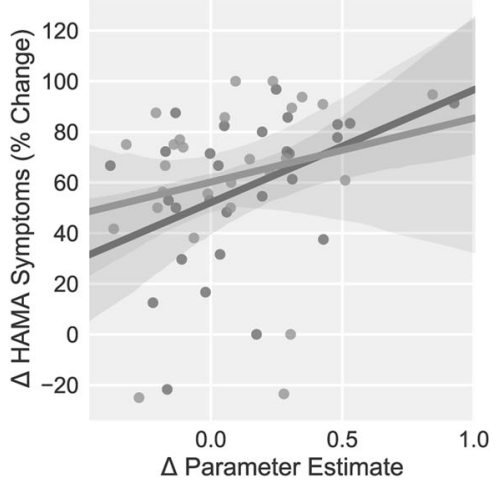

\section{B.i. Treatment Prediction: Depression}

\section{Emotion Perception: Insula}

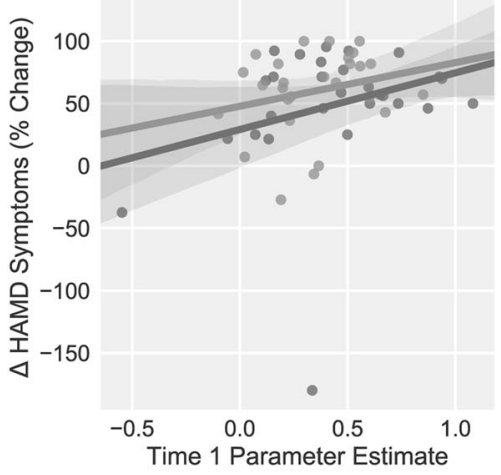

Emotion Perception: Amygdala

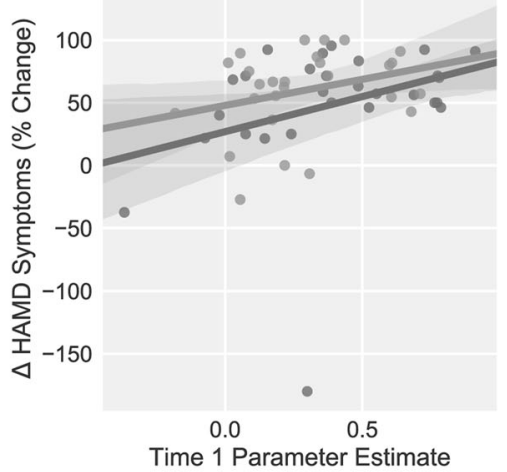

\section{B.ii. Treatment Prediction: Anxiety}

\section{Emotion Perception: Insula}

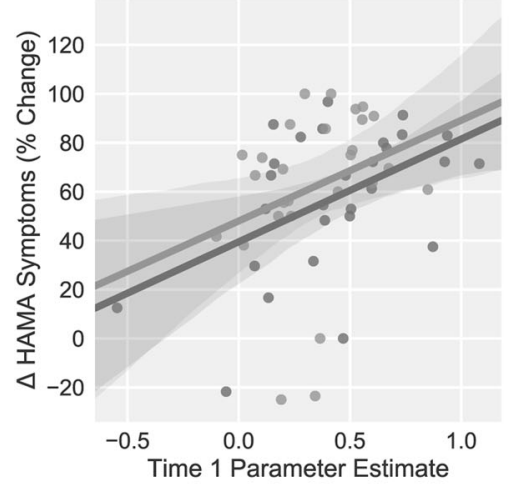

\section{Emotion Perception: Amygdala}

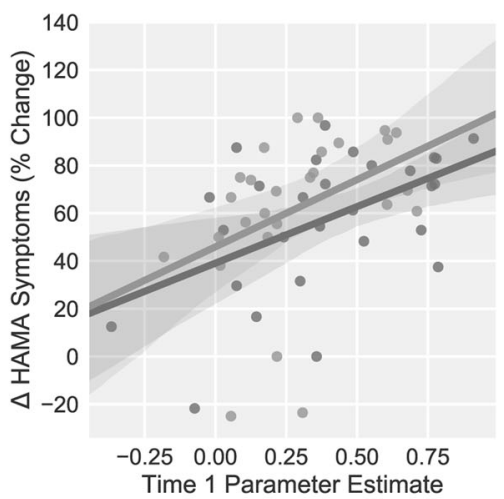

Treatment Arm

SSRI $(n=28)$

$$
-*-\operatorname{CBT}(n=27)
$$

Fig. 3 Relation between symptom improvement and brain activation. a Greater anxiety improvement, as measured by the Hamilton Rating Scale for Anxiety (HAMA) total score, was significantly correlated with greater decreases pre-treatment to post-treatment in insula (left) and amygdala (right) activation during emotion perception. b.i Greater depression symptom improvement, as measured by the Hamilton Rating Scale for Depression (HAMD) total score, was associated with greater pre-treatment anterior insula (left) and amygdala (middle) activation during emotion perception. b.ii Greater anxiety symptom improvement, as measured by the HAMA total, was also associated with greater pretreatment insula (left) and amygdala (right) activation during emotion perception. Shaded region represents bootstrapped $95 \%$ confidence intervals 
arm and thus, it is possible that the current findings reflect general effects of time or naturalistic symptom reduction rather than treatment-specific changes in brain activation. Feasibility and difficulty of a study design that involved two kinds of placebo for the two treatments precluded a more rigorous test of treatmentmediated vs. non-specific effects. Of note, because of the randomized design that is similar to recent studies with similar aims [19], there is no reason to expect different rates of placebo response between the two treatments. Second, though 122 participants were randomized to treatment, only 77 participants completed the post-treatment scan of which 55 participants had usable pre-treatment and post-treatment fMRI data. Thus, there was considerable dropout and failure to return for the posttreatment scan, which is the nature of a 12-week treatment study with a second fMRI session. Third, the study only included two assessment points. It is useful to understand when brain change is detectable during treatment, whether treatment-related brain changes are sustained and whether neural markers can predict course of symptoms beyond immediate treatment termination. Fourth, our participant sample was primarily female and had high levels of comorbidity, which increases external validity, but also prohibits us from determining the impact of sex and specific diagnoses on the pattern of results. It is also important to note that mean levels of depression and anxiety symptoms at baseline were mild and it is unclear whether the findings generalize to more severe clinical samples. Fifth, several other factors that were not tested in the present study may have influenced treatment outcomes and the pattern of results including variability in SSRI dosing and medication type [42, 43], and personality [44]. Sixth, we utilized two complementary tasks of emotion processing, but there are several other tasks that were not included but are relevant to SSRI and CBT treatment. Relatedly, we only examined ROls that were probed by our tasks, and there may be other important regions not captured by our measures that are related to internalizing psychopathology treatment outcomes. We also examined anatomical ROls and did not test whether different subregions within our ROls yielded different patterns of results. For instance, the posterior and anterior insula differ in their core functions such that the posterior insula receives and integrates somatosensory information while the anterior insula underlies the subjective evaluation of internal conditions [45]. It is possible that these two subregions, as well as others, are differentially involved in treatment mechanisms and prediction and should be considered in future studies.

The current study identifies shared, focal neural processes and predictors of response across the two first-line internalizing psychopathology treatments. These findings help refine our understanding of internalizing psychopathology treatments, and demonstrate that $\mathrm{fMRI}$ assays of emotion perception may reflect treatment mechanisms and can be used as predictors of treatment response.

\section{FUNDING AND DISCLOSURE}

This study was funded by the National Institute of Mental Health of the National Institutes of Health grant R01MH101497 (to KLP) and the National Center for Advancing Translational Sciences, National Institutes of Health, through grant UL1TR002003. SMG is funded by the National Institute on Alcohol Abuse and Alcoholism grant K23AA025111. MBS has in the past 3 years been a consultant for Actelion, Aptinyx, Bionomics, Dart Neuroscience, Healthcare Management Technologies, Janssen, Neurocrine Biosciences, Oxeia Biopharmaceuticals, Pfizer, and Resilience Therapeutics. MBS owns founders shares and stock options in Resilience Therapeutics and has stock options in Oxeia Biopharmaceuticals. The remaining authors declare that they have no conflict of interest.

\section{ADDITIONAL INFORMATION}

Supplementary Information accompanies this paper at (https://doi.org/10.1038/ s41386-019-0407-7)

Publisher's note: Springer Nature remains neutral with regard to jurisdictional claims in published maps and institutional affiliations.

\section{REFERENCES}

1. Demyttenaere K, Bruffaerts R, Posada-Villa J, Gasquet I, Kovess V, Lepine JP, et al. Prevalence, severity, and unmet need for treatment of mental disorders in the World Health Organization World Mental Health Surveys. JAMA. 2004;291:2581-90.

2. DeRubeis RJ, Siegle GJ, Hollon SD. Cognitive therapy vs. medications for depression: treatment outcomes and neural mechanisms. Nat Rev Neurosci. 2008:9:788-96.

3. Amick HR, Gartlehner G, Gaynes BN, Forneris C, Asher GN, Morgan LC, et al. Comparative benefits and harms of second generation antidepressants and cognitive behavioral therapies in initial treatment of major depressive disorder: systematic review and meta-analysis. BMJ. 2015;351:h6019.

4. Papakostas GI, Fava M, Thase ME. Treatment of SSRI-resistant depression: a metaanalysis comparing within- versus across-class switches. Biol Psychiatry. 2008:63:699-704.

5. Loerinc AG, Meuret AE, Twohig MP, Rosenfield D, Bluett EJ, Craske MG. Response rates for $\mathrm{CBT}$ for anxiety disorders: need for standardized criteria. Clin Psychol Rev. 2015;42:72-82.

6. Pico-Perez M, Radua J, Steward T, Menchon JM, Soriano-Mas C. Emotion regulation in mood and anxiety disorders: a meta-analysis of $\mathrm{fMRI}$ cognitive reappraisal studies. Prog Neuro-Psychopharmacol Biol psychiatry. 2017;79:96-104.

7. Lindquist KA, Wager TD, Kober H, Bliss-Moreau E, Barrett LF. The brain basis of emotion: a meta-analytic review. Behav Brain Sci. 2012;35:121-43.

8. Buhle JT, Silvers JA, Wager TD, Lopez R, Onyemekwu C, Kober H, et al. Cognitive reappraisal of emotion: a meta-analysis of human neuroimaging studies. Cereb Cortex. 2014;24:2981-90.

9. Groenewold NA, Opmeer EM, de Jonge P, Aleman A, Costafreda SG. Emotional valence modulates brain functional abnormalities in depression: evidence from a meta-analysis of fMRI studies. Neurosci Biobehav Rev. 2013;37:152-63.

10. MacNamara A, Klumpp $H$, Kennedy AE, Langenecker SA, Phan KL. Transdiagnostic neural correlates of affective face processing in anxiety and depression. Depress Anxiety. 2017:34:621-31.

11. Ma Y. Neuropsychological mechanism underlying antidepressant effect: a systematic meta-analysis. Mol Psychiatry. 2015;20:311-9.

12. Brooks SJ, Stein DJ. A systematic review of the neural bases of psychotherapy for anxiety and related disorders. Dialog Clin Neurosci. 2015;17:261-79.

13. Klumpp H, Fitzgerald DA, Phan KL. Neural predictors and mechanisms of cognitive behavioral therapy on threat processing in social anxiety disorder. Prog Neuro-Psychopharmacol Biol Psychiatry. 2013;45:83-91.

14. Young KS, Burklund LJ, Torre JB, Saxbe D, Lieberman MD, Craske MG. Treatment for social anxiety disorder alters functional connectivity in emotion regulation neural circuitry. Psychiatry Res Neuroimaging. 2017;261:44-51.

15. Burklund L, Torre JB, Lieberman MD, Taylor SE, Craske MG. Neural responses to social threat and predictors of cognitive behavioral therapy and acceptance and commitment therapy in social anxiety disorder. Psychiatry Res Neuroimaging 2017:261:52-64.

16. Fu CH, Steiner $\mathrm{H}$, Costafreda SG. Predictive neural biomarkers of clinical response in depression: a meta-analysis of functional and structural neuroimaging studies of pharmacological and psychological therapies. Neurobiol Dis. 2013;52:75-83.

17. Siegle GJ, Carter CS, Thase ME. Use of FMRI to predict recovery from unipolar depression with cognitive behavior therapy. Am J Psychiatry. 2006;163:735-8.

18. Whalen PJ, Johnstone $T$, Somerville $L H$, Nitschke JB, Polis $S$, Alexander AL, et al. A functional magnetic resonance imaging predictor of treatment response to venlafaxine in generalized anxiety disorder. Biol Psychiatry. 2008;63:858-63.

19. Dunlop BW, Binder EB, Cubells JF, Goodman MM, Kelley ME, Kinkead B, et al. Predictors of remission in depression to individual and combined treatments (PReDICT): study protocol for a randomized controlled trial. Trials. 2012;13:106.

20. Lovibond SH, Lovibond PF. Manual for the depression anxiety stress scale. 2nd ed. Sydney: Psychology Foundation; 1995

21. First MB, Williams JBW, Karg RS, Spitzer RL. Structured Clinical Interview for DSM5-Research Version (SCID-5 for DSM-5, Research Version; SCID-5-RV). Arlington, VA: American Psychiatric Association; 2015.

22. Hamilton M. A rating scale for depression. J Neurol Neurosurg Psychiatry. 1960;23:56-62.

23. Hamilton $\mathrm{M}$. The assessment of anxiety states by rating. $\mathrm{Br} J$ Med Psychol. 1959:32:50-5. 
1648

24. Nicolini H, Bakish D, Duenas H, Spann M, Erickson J, Hallberg C, et al. Improvement of psychic and somatic symptoms in adult patients with generalized anxiety disorder: examination from a duloxetine, venlafaxine extended-release and placebo-controlled trial. Psychol Med. 2009;39:267-76.

25. Gorka SM, Burkhouse KL, Klumpp H, Kennedy AE, Afshar K, Francis J, et al. Errorrelated brain activity as a treatment moderator and index of symptom change during cognitive-behavioral therapy or selective serotonin reuptake inhibitors. Neuropsychopharmacology. 2017;43:1355.

26. Burkhouse KL, Gorka SM, Klumpp H, Kennedy AE, Karich S, Francis J, et al. Neural responsiveness to reward as an index of depressive symptom change following cognitive-behavioral therapy and SSRI treatment. J Clin Psychiatry. 2018;79:pii: $17 \mathrm{~m} 11836$.

27. Fusar-Poli $P$, Placentino A, Carletti F, Landi $P$, Allen $P$, Surguladze $S$, et al. Functional atlas of emotional faces processing: a voxel-based meta-analysis of 105 functional magnetic resonance imaging studies. J Psychiatry Neurosci. 2009;34:418-32.

28. Yarkoni T, Poldrack RA, Nichols TE, Van Essen DC, Wager TD. Large-scale automated synthesis of human functional neuroimaging data. Nat methods. 2011;8:665-70.

29. Fu CH, Williams SC, Cleare AJ, Brammer MJ, Walsh ND, Kim J, et al. Attenuation of the neural response to sad faces in major depression by antidepressant treatment: a prospective, event-related functional magnetic resonance imaging study. Arch Gen Psychiatry. 2004;61:877-89.

30. Fonzo GA, Ramsawh HJ, Flagan TM, Sullivan SG, Simmons AN, Paulus MP, et al. Cognitive-behavioral therapy for generalized anxiety disorder is associated with attenuation of limbic activation to threat-related facial emotions. J Affect Disord. 2014;169:76-85.

31. Simmons AN, Arce E, Lovero KL, Stein MB, Paulus MP. Subchronic SSRI administration reduces insula response during affective anticipation in healthy volunteers. Int J Neuropsychopharmacol. 2009;12:1009-20.

32. Menon V, Uddin LQ. Saliency, switching, attention and control: a network model of insula function. Brain Struct Funct. 2010;214:655-67.

33. Menon V. Large-scale brain networks and psychopathology: a unifying triple network model. Trends Cogn Sci. 2011;15:483-506.

34. Uddin LQ. Salience processing and insular cortical function and dysfunction. Nat Rev Neurosci. 2015;16:55-61.
35. Etkin A, Wager TD. Functional neuroimaging of anxiety: a meta-analysis of emotional processing in PTSD, social anxiety disorder, and specific phobia. Am J Psychiatry. 2007;164:1476-88.

36. Klumpp H, Fitzgerald JM. Neuroimaging predictors and mechanisms of treatment response in social anxiety disorder: an overview of the amygdala. Curr Psychiatry Rep. 2018;20:89.

37. Fonseka TM, MacQueen GM, Kennedy SH. Neuroimaging biomarkers as predictors of treatment outcome in major depressive disorder. J Affect Disord. 2018:233:21-35

38. Niles AN, Mesri B, Burklund LJ, Lieberman MD, Craske MG. Attentional bias and emotional reactivity as predictors and moderators of behavioral treatment for social phobia. Behav Res Ther. 2013;51:669-79.

39. MacNamara $A$, Rabinak $C A$, Kennedy $A E$, Fitzgerald $D A$, Liberzon I, Stein $M B$, et al. Emotion regulatory brain function and SSRI treatment in PTSD: neural correlates and predictors of change. Neuropsychopharmacology. 2015;41:611

40. Goldapple K, Segal Z, Garson C, Lau M, Bieling P, Kennedy S, et al. Modulation of cortical-limbic pathways in major depression: treatment-specific effects of cognitive behavior therapy. Arch Gen Psychiatry. 2004;61:34-41.

41. Zald DH, Lahey BB. Implications of the hierarchical structure of psychopathology for psychiatric neuroimaging. Biol Psychiatry Cogn Neurosci Neuroimaging. 2017;2:310-7.

42. Jakubovski E, Varigonda AL, Freemantle N, Taylor MJ, Bloch MH. Systematic review and meta-analysis: dose-response relationship of selective serotonin reuptake inhibitors in major depressive disorder. Am J Psychiatry. 2015;173:174-83.

43. Jakubovski E, Johnson JA, Nasir M, Müller-Vahl K, Bloch MH. Systematic review and meta-analysis: dose-response curve of SSRIs and SNRIs in anxiety disorders. Depress Anxiety. 2019;36:198-212.

44. Wolitzky-Taylor KB, Arch JJ, Rosenfield D, Craske MG. Moderators and nonspecific predictors of treatment outcome for anxiety disorders: a comparison of cognitive behavioral therapy to acceptance and commitment therapy. J Consult Clin Psychol. 2012;80:786-99.

45. Craig AD. How do you feel-now? The anterior insula and human awareness. Nat Rev Neurosci. 2009;10:59-70. 